\title{
Risk factors for early pulmonary valve replacement after valve disruption in congenital pulmonary stenosis and tetralogy of Fallot
}

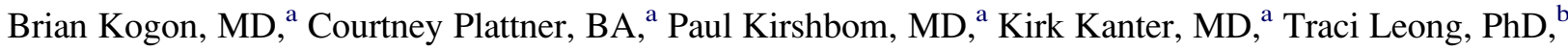 \\ Theresa Lyle, NPN, ${ }^{\mathrm{a}}$ Staci Jennings, RN, ${ }^{\mathrm{a}}$ Mike McConnell, MD, ${ }^{\mathrm{c}}$ and Wendy Book, $\mathrm{MD}^{\mathrm{a}}$
}

\begin{abstract}
Objective: Congenital heart defects with a component of pulmonary stenosis are often palliated in childhood by disrupting the pulmonary valve, either by means of dilation or excision. It is unclear what factors affect a patient's ability to tolerate long-term pulmonary insufficiency before requiring pulmonary valve replacement. We analyze potential factors that are related to the interval between pulmonary valve disruption and pulmonary valve replacement.
\end{abstract}

\begin{abstract}
Methods: One hundred seven patients were analyzed. They had a congenital diagnosis of pulmonary stenosis or tetralogy of Fallot, had their first pulmonary valve replacement between 2002 and 2008, and had a known interval between pulmonary valve disruption and pulmonary valve replacement. The median age at the time of surgical intervention was 2 years for pulmonary valve disruption (range, 0-56 years) and 26 years for pulmonary valve replacement (range, 1-72 years). The median interval was 23 years (range, $0-51$ years). Potential related factors were sex, race, initial diagnosis and procedure, age at pulmonary valve disruption, prior shunt operation, presence of branch pulmonary artery stenosis, and degree of pulmonary regurgitation.

Results: As determined by using univariate analysis, male patients had a shorter interval than female patients (median, 16 vs 26 years; $P=.01$ ), and African American patients had a shorter interval than white patients (median, 16 vs 25 years; $P=.049$ ). A significant correlation was also identified between age at the time of pulmonary valve disruption and the subsequent interval to pulmonary valve replacement. Overall, the interval tended to increase as age at disruption increased $(P<.0001)$. Although the presence of branch pulmonary artery stenosis determined by the need for concomitant pulmonary arterioplasty was associated with a significantly shorter interval to pulmonary valve replacement ( 21 vs 24 years, $P=.02$ ), stenosis determined based on small branch pulmonary artery diameter was correlated to a prolonged interval to pulmonary valve replacement $(\mathrm{P}=.009)$. Initial diagnosis, prior palliative shunt operation, and degree of pulmonary regurgitation had no effect on the interval between pulmonary valve disruption and subsequent pulmonary valve replacement. As determined by using multivariate analysis, only male sex and small pulmonary artery diameter remained significant factors.
\end{abstract}

Conclusions: Male sex appears to shorten the interval between pulmonary valve disruption and pulmonary valve replacement, whereas small branch pulmonary artery diameter appears to lengthen the interval. Knowing which factors are detrimental and which are protective might help identify patients who are prone to a more rapid progression of right heart failure from free pulmonary insufficiency, possibly steering them toward more frequent follow-up or more aggressive heart failure medical regimens.

Surgical management of tetralogy of Fallot and congenital pulmonary stenosis often results in anatomic and functional abnormalities: an absent pulmonary valve and free pulmonary insufficiency. Although the right ventricular (RV) volume load caused by severe pulmonary insufficiency can be tolerated for years, there is now evidence that the compensatory mechanisms of the RV myocardium will gradually fail. ${ }^{1}$

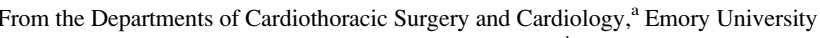
School of Medicine; the Rollins School of Public Health ${ }^{\text {b }}$, and Sibley Heart Center Cardiology, ${ }^{\mathrm{c}}$ Children's Healthcare of Atlanta, Atlanta, Ga.

Received for publication Feb 6, 2008; revisions received Dec 11, 2008; accepted for publication Feb 2, 2009.

Address for reprints: Brian Kogon, MD, Cardiothoracic Surgery, Emory University, 1405 Clifton Rd NE, Atlanta, GA 30322 (E-mail: Brian_kogon@emoryhealthcare. org).

J Thorac Cardiovasc Surg 2009;138:103-8

$0022-5223 / \$ 36.00$

Copyright (c) 2009 by The American Association for Thoracic Surgery

doi:10.1016/j.jtcvs.2009.02.020
}

It is unclear what factors affect a patient's ability to tolerate long-term pulmonary insufficiency before requiring pulmonary valve replacement.

\section{MATERIALS AND METHODS}

After internal review board approval was obtained, patients who underwent pulmonary valve replacement were reviewed. They were included in the analysis if they had a congenital diagnosis of pulmonary stenosis or tetralogy of Fallot, if their initial pulmonary valve replacement occurred between the years 2002 and 2006, and if they had a known interval between initial valve disruption and valve replacement.

\section{Population Description}

Patients had an initial diagnosis of tetralogy of Fallot $(n=90)$ and congenital pulmonary stenosis $(\mathrm{n}=17)$. Before the valve-disrupting operation, 29 patients underwent 26 palliative shunting operations. These shunts included Blalock-Taussig shunts $(n=24)$, Potts shunts $(n=4)$, and Waterston shunts $(\mathrm{n}=1)$. The exact type of valve-disrupting operation was 


\section{Abbreviations and Acronyms \\ CMR = cardiac magnetic resonance imaging \\ $\mathrm{LV}=$ left ventricular \\ PA/BSA $=$ smallest diameter of branch pulmonary artery identified and indexed to body surface area \\ RV

$$
=\text { right ventricular }
$$

difficult to obtain from old records. With resultant pulmonary insufficiency, indications for valve replacement surgery included the presence of symptoms, decreased RV function, decreased left ventricular (LV) function, $\mathrm{RV} / \mathrm{LV}$ end-diastolic volume ratio of greater than 2, QRS duration of greater than $180 \mathrm{~ms}$, or progressive tricuspid regurgitation (Figure 1). One hundred three patients received bioprosthetic valves, and 4 received mechanical valves (Figure 2). Sixty concomitant procedures were performed in 44 patients (Figure 3). The most common concomitant operations were tricuspid valve surgery and branch pulmonary arterioplasty (11 on the left and 4 on the right). No patients have required subsequent pulmonary valve rereplacement. There were 3 surgical mortalities: 1 in a high-risk patient with multiple comorbidities, another in a patient requiring concomitant 3-vessel coronary bypass, and a third in a patient requiring concomitant aortic valve and root replacement.

\section{Imaging}

Preoperative imaging studies included echocardiography, cardiac catheterization, and cardiac magnetic resonance imaging (CMR). Fifty-eight patients underwent preoperative CMR. Parameters analyzed were ventricular chamber size and ratio, ejection fraction, pulmonary regurgitant fraction, and branch pulmonary artery diameter (Table 1). The smallest branch pulmonary artery diameter was divided by the patient's body surface area to obtain the smallest branch pulmonary artery index. Chamber measurements revealed a median RV end-diastolic volume index of $148 \mathrm{~mL} / \mathrm{m}^{2}$ (range, 74-308 $\mathrm{mL} / \mathrm{m}^{2}$ ), an $\mathrm{RV}$ end-systolic volume index of $82 \mathrm{~mL} / \mathrm{m}^{2}$ (range, $\left.28-195 \mathrm{~mL} / \mathrm{m}^{2}\right)$, and an $\mathrm{LV}$ end-diastolic volume index of $69 \mathrm{~mL} / \mathrm{m}^{2}$ (range, $38-147 \mathrm{~mL} / \mathrm{m}^{2}$ ). The median $\mathrm{RV} / \mathrm{LV}$ end-diastolic volume ratio was 1.98 (range, 0.94-3.5). Functional measurements revealed a median RV ejection fraction of $45 \%$ (range, $24 \%-52 \%$ ) and an LV ejection fraction of $59 \%$ (range, $29 \%-65 \%$ ). The median pulmonary regurgitant fraction was $39 \%$ (range, $10 \%-76 \%$ ). The median smallest branch pulmonary artery diameter index was $8.8 \mathrm{~mm} / \mathrm{m}^{2}$ (range, $1.4-21.6 \mathrm{~mm} / \mathrm{m}^{2}$ ).

The Mann-Whitney test was used to compare 2 groups with respect to a continuous end point, such as the interval to pulmonary valve replacement. Binary variables included sex, race, presence of branch pulmonary artery stenosis (determined by concomitant pulmonary arterioplasty at the time of pulmonary valve replacement), diagnosis, and history of a prior palliative shunt.

Spearman correlation coefficients were calculated to assess the relationship between continuous variables and interval to pulmonary valve replacement. Continuous variables included age, the smallest branch pulmonary artery determined by using CMR, and regurgitant fraction determined by using CMR.

A multiple regression model was then used to further evaluate those factors with a significant relationship to the interval between pulmonary valve disruption and pulmonary valve replacement.

\section{RESULTS}

One hundred seven patients met the study criteria and were included in the analysis.

Regarding sex, 47 patients were male, and 60 patients were female. Male patients had a significantly shorter inter-

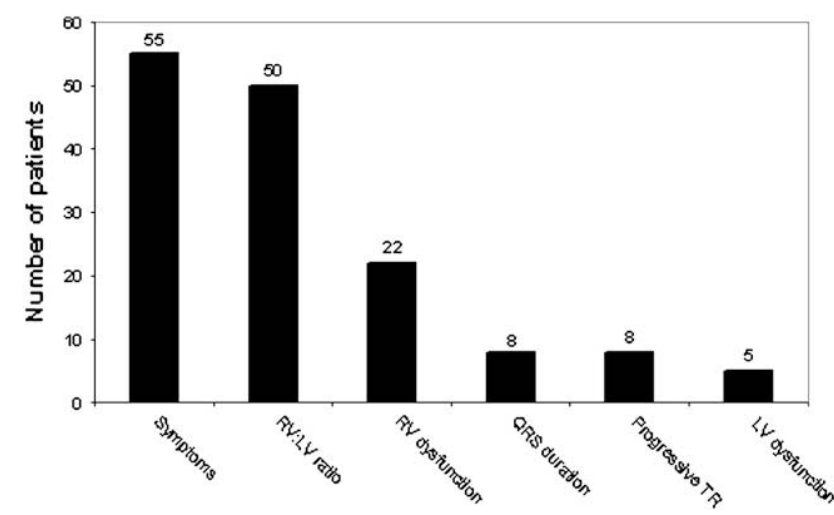

FIGURE 1. Indications for pulmonary valve replacement. $R V$, Right ventricular; $L V$, left ventricular; $T R$, tricuspid regurgitation.

val to pulmonary valve replacement than female patients (median, 16 vs 26 years; $P=.009$; Figure 4 ).

Regarding race, 23 patients were African American, 82 were white, and 2 were of other racial backgrounds. African American patients had a shorter interval to pulmonary valve replacement than white patients (median, 16 vs 25 years; $P=.049 ;$ Figure 5).

Regarding age, the median age at the time of surgical intervention was 2 years for pulmonary valve disruption (range, $0-56$ years) and 26 years for pulmonary valve replacement (range, 1-72 years), resulting in a median interval between pulmonary valve disruption and replacement of 22 years (range, 0-47). A significant correlation was identified between age at the time of disruption and the subsequent interval to pulmonary valve replacement. Overall, the pulmonary valve replacement interval tended to increase as age at disruption increased $(P<.0001)$.

The presence of branch pulmonary artery stenosis was initially determined by the need for concomitant pulmonary arterioplasty at the time of pulmonary valve replacement and by means of preoperative CMR. During pulmonary valve replacement, 13 patients underwent pulmonary arterioplasty. By using these criteria, branch pulmonary artery stenosis was associated with a significantly shorter interval to pulmonary valve replacement ( 21 vs 24 years, $P=.02$, Figure 6). By using the preoperative magnetic resonance imaging, the smallest diameter of the branch pulmonary artery was identified and indexed to the body surface area (PA/BSA). There was a significant correlation between PA/BSA and the interval to pulmonary valve replacement. However, conversely, as the size of the branch pulmonary arteries decreased, the interval to pulmonary valve replacement increased $(P=.009)$.

Regarding surgical history, 26 patients underwent a systemic-pulmonary artery shunt before their valve-disrupting procedures. Placement of a shunt before the initial valve disruption had no effect on the interval between subsequent pulmonary valve disruption and pulmonary valve replacement $(P=.70)$. 


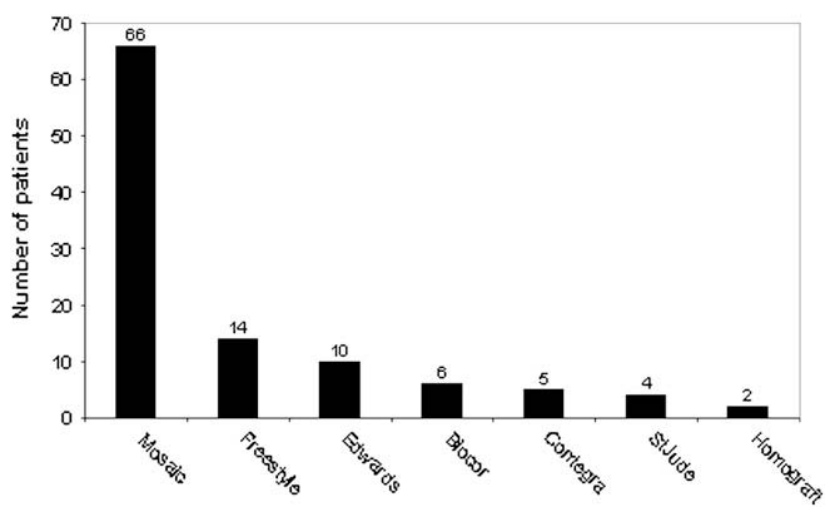

FIGURE 2. Pulmonary valve replacement types.

In addition, there was no relationship between the diagnosis and interval to pulmonary valve replacement. Both groups had a median interval of 23 years (range, $0-51$ years for tetralogy of Fallot and 3-47 years for pulmonary stenosis). There was also no correlation between the regurgitant fraction and the interval to pulmonary valve replacement.

A multivariate regression analysis was performed for sex, race, age at pulmonary valve disruption, presence of branch pulmonary stenosis determined by means of concomitant pulmonary artery plasty, and smallest PA/BSA determined by means of CMR. Risk factors maintaining significance were sex and PA/BSA (Table 2).

\section{DISCUSSION}

\section{Evaluation}

Clinical history, physical examination, electrocardiography, and echocardiography are all useful tools in evaluating pulmonary insufficiency. However, CMR has become the gold standard imaging modality for the periodic evaluation and follow-up of patients with pulmonary regurgitation. Systolic and diastolic flow through the pulmonary valve

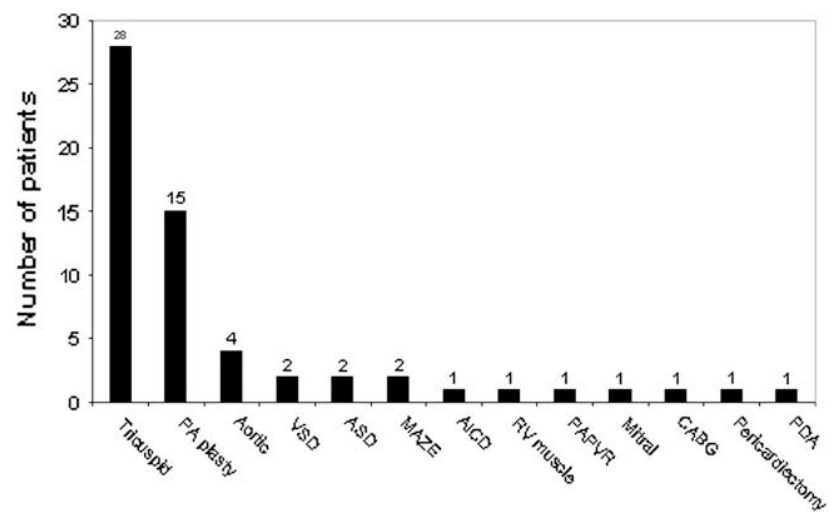

FIGURE 3. Concomitant operations. $P A$, Pulmonary artery; $V S D$, ventricular septal defect; $A S D$, atrial septal defect; $A I C D$, automatic implantable cardioverter defibrillator; $R V$, right ventricular; $P A P V R$, partial anomalous pulmonary venous return; $C A B G$, coronary artery bypass grafting; $P D A$, patent ductus arteriosus.
TABLE 1. Summary of CMR data

\begin{tabular}{lcc}
\hline & Median & Range \\
\hline RV end-diastolic volume index $\left(\mathrm{mL} / \mathrm{m}^{2}\right)$ & 148 & $74-308$ \\
RV end-systolic volume index $\left(\mathrm{mL} / \mathrm{m}^{2}\right)$ & 82 & $28-195$ \\
LV end-diastolic volume index $\left(\mathrm{mL} / \mathrm{m}^{2}\right)$ & 69 & $38-147$ \\
RV/LV end-diastolic volume ratio & 1.98 & $0.94-3.5$ \\
RV ejection fraction (\%) & 45 & $24-52$ \\
LV ejection fraction (\%) & 59 & $29-65$ \\
Regurgitant fraction $(\%)$ & 39 & $10-76$ \\
Smallest branch pulmonary artery index $\left(\mathrm{mm} / \mathrm{m}^{2}\right)$ & 8.8 & $1.4-21.6$ \\
\hline$C M R$, Cardiac magnetic resonance imaging; $R V$, right ventricular; $L V$, left ventricular.
\end{tabular}

can be quantified, allowing for calculation of pulmonary regurgitant fraction. Unlike echocardiography, CMR works independent of geometric assumptions for evaluation of $\mathrm{RV}$ volume and function and can better assess the branch pulmonary artery anatomy. Overall, CMR provides essential and complementary data, facilitating management and prognosis for these patients. ${ }^{2}$

All of our patients underwent a complete clinical history and physical examination, electrocardiography, and echocardiography. Potential surgical candidates more than 40 years of age underwent cardiac catheterization for coronary angiography. Although magnetic resonance imaging is the diagnostic modality advocated in these patients and our recent trend is for all of patients to undergo CMR, we have not yet achieved that goal. The patients who did not undergo CMR before pulmonary valve replacement were primarily earlier in the study period, our younger patients in whom CMR would be difficult or would require sedation/intubation, those with medical devices (pacemakers, coils, stents) in whom CMR is limited or contraindicated, and those who met indications for surgical intervention without CMR whose pulmonary artery anatomy was previously delineated by means of cardiac catheterization.

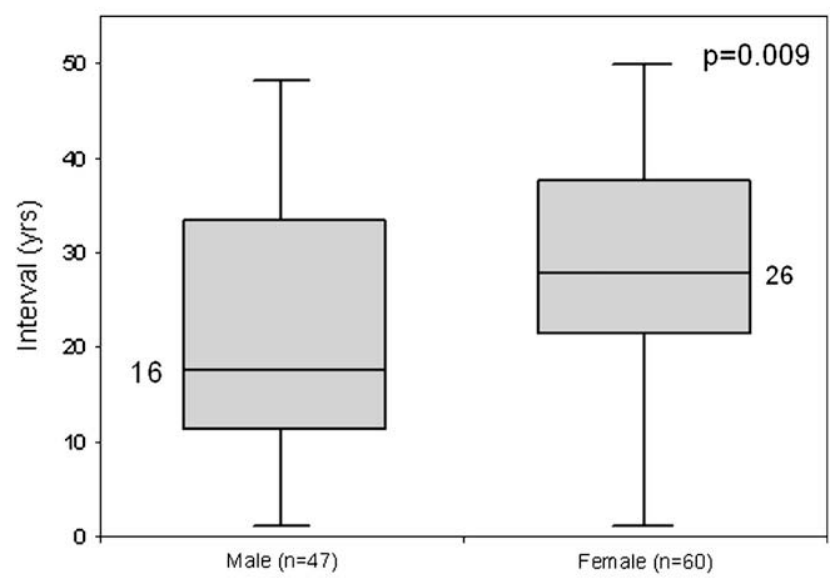

FIGURE 4. Relationship between sex and interval to pulmonary valve replacement. 


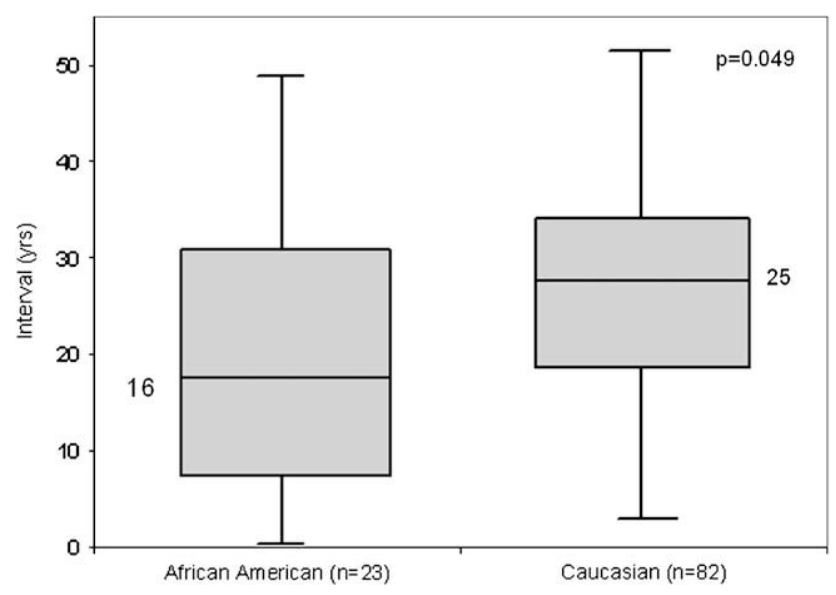

FIGURE 5. Relationship between race and interval to pulmonary valve replacement.

\section{Indications for Surgical Intervention}

Although the optimal timing of surgical intervention remains a challenge and decisions need to be made on an individual basis, pulmonary valve replacement should be performed in these patients before the development of irreversible ventricular dysfunction. Some programs have set very specific recommendations for pulmonary valve replacement in this patient population. These include repaired tetralogy of Fallot or similar physiology with moderate or severe pulmonary regurgitation (regurgitant fraction $>25 \%$, as measured with CMR) and 2 or more of the following criteria: (1) RV end-diastolic volume index of $160 \mathrm{~mL} / \mathrm{m}^{2}$ or greater, $\mathrm{RV}$ end-systolic volume index of $70 \mathrm{~mL} / \mathrm{m}^{2}$ or greater, $\mathrm{LV}$ end-diastolic volume index of $65 \mathrm{~mL} / \mathrm{m}^{2}$ or less, $\mathrm{RV}$ ejection fraction of $45 \%$ or less, RV outflow tract aneurysm, and clinical criteria, such as exercise intolerance, symptoms and signs of heart failure, cardiac medications, syncope, and sustained ventricular tachycardia. ${ }^{1}$ The presence of

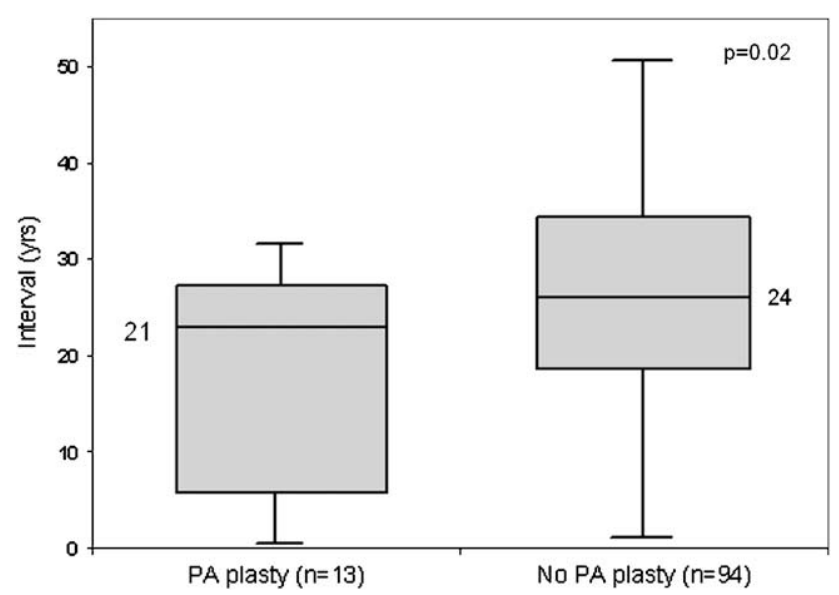

FIGURE 6. Relationship between pulmonary artery $(P A)$ plasty and interval to pulmonary valve replacement.
TABLE 2. Multivariate risk factor analysis for shorter interval to pulmonary valve replacement

\begin{tabular}{|c|c|c|c|c|c|}
\hline Characteristic & No. & $\begin{array}{c}\text { Mean } \\
\text { interval }(y)\end{array}$ & $\begin{array}{c}\text { Median } \\
\text { interval }(y)\end{array}$ & Correlation & $\begin{array}{c}P \\
\text { value }\end{array}$ \\
\hline \multicolumn{6}{|l|}{ Male sex } \\
\hline Yes & 47 & 19.3 & 16 & & (+) .003 \\
\hline No & 60 & 25.6 & 26 & & \\
\hline \multicolumn{6}{|l|}{ African American } \\
\hline Yes & 23 & 18.4 & 16 & & .806 \\
\hline No & 82 & 24.3 & 25 & & \\
\hline \multicolumn{6}{|l|}{$\begin{array}{c}\text { Concomitant branch } \\
\text { PA plasty }\end{array}$} \\
\hline Yes & 13 & 15.2 & 21 & & .902 \\
\hline No & 94 & 23.9 & 24 & & \\
\hline $\begin{array}{l}\text { Smallest branch } \\
\text { PA index }\end{array}$ & & & & (-) 0.412 & $(-) .013$ \\
\hline $\begin{array}{l}\text { Increased age at } \\
\text { pulmonary valve } \\
\text { disruption }\end{array}$ & & & & 0.3176 & .324 \\
\hline
\end{tabular}

other hemodynamically significant lesions, such as moderate-to-severe tricuspid regurgitation, residual atrial or ventricular septal defects, and severe aortic insufficiency, also triggered referral for surgical intervention in patients with moderate or severe pulmonary regurgitation. ${ }^{1}$

We have been relatively aggressive at recommending pulmonary valve replacement in these patients, and our criteria are quite similar to those reported in the literature. They have remained consistent over the study period and include the presence of symptoms, decreased RV function, decreased $\mathrm{LV}$ function, $\mathrm{RV} / \mathrm{LV}$ end-diastolic volume ratio of greater than 2, QRS duration of greater than $180 \mathrm{~ms}$, and progressive tricuspid regurgitation.

\section{Risk Factors}

Regarding sex and race, because patient outcomes are highly variable despite similar anatomy and hemodynamics, genetic differences might affect a patient's ability to tolerate long-term pulmonary insufficiency before right heart failure develops and requirement for pulmonary valve replacement. The interaction between genetic variability and outcome has been well studied in adult cardiac disease, and common genetic variants in candidate genes of the cardiovascular system are likely to play a role in the development of heart failure, its phenotypic evolution, and, more importantly, its response to therapeutic intervention. ${ }^{3,4}$ Although sex differences remained significant in the univariate and multivariate analysis, racial differences were barely significant in only the univariate analysis. Studying the genetic variability in these patients might be an important area for future research. Additionally, evaluation of differences in the RV myocardium or pulmonary vasculature between sexes and races might elucidate some answers as well. 
Regarding pulmonary stenosis, it has long been known, anecdotally, that a combination of severe distal pulmonary artery stenosis and a transannular patch leads to early RV dilation and failure. ${ }^{5} \mathrm{RV}$ pressure-volume relationships show a linear increase in pulmonary regurgitant volume as RV afterload increases. ${ }^{6}$ Our analysis showed a significant relationship between branch pulmonary artery stenosis defined by performing a concomitant arterioplasty at the time of pulmonary valve replacement and a shorter interval to pulmonary valve replacement. Unfortunately, the decision to perform an arterioplasty was not always related to objective measurements of branch pulmonary artery gradients or sizes, and the decision was sometimes made intraoperatively based solely on branch pulmonary artery appearance. This factor did not remain significant in the multivariate analysis. On the other hand, pulmonary artery stenosis defined objectively based on branch pulmonary artery size had the opposite effect. It not only prolonged the interval to pulmonary valve replacement but remained significant in both univariate and multivariate analyses. By using the smallest branch pulmonary artery diameter measured by means of CMR and indexing it to body surface area, a significant correlation was identified between PA/BSA and a longer interval to pulmonary valve replacement. Interestingly, there was no relationship between the regurgitant fraction and the interval to pulmonary valve replacement.

Although it does indeed seem counterintuitive that pulmonary artery stenosis would somehow be protective and delay the interval to pulmonary valve replacement, investigation of the RV myocardium might provide insight into this issue. It is unclear whether there are differences in the neonatal and adult RV myocardium, and it is unclear whether changes in the right ventricle that occur from some degree of pulmonary stenosis (hypertrophy, increased stiffness, and decreased compliance) might be protective against the changes that occur from regurgitation.

For those patients who have some degree of residual pulmonary artery stenosis, it has been suggested that it should be relieved by balloon dilation, stent implantation, or surgical intervention if one is to sustain RV function in the very long term. ${ }^{7,8}$ However, again it might be that some degree of pulmonary stenosis (small pulmonary arteries) could result in changes in the right ventricle that are protective against RV dilation and the progression of RV failure.

Studies have also shown that surrogates of increased pulmonary vascular resistance (eg, Waterston or Potts shunts) are also associated with increased adverse effects of pulmonary regurgitation. ${ }^{9}$ Our analysis did not identify a relationship between prior systemic-pulmonary artery shunt and interval to pulmonary valve replacement. Nonetheless, with early primary repair, these effects are less likely to be seen in the current era.

Regarding age at disruption, many patients have no significant distal pulmonary artery stenosis and no reason for an increased pulmonary arteriolar resistance. Likely, there is a role of the right ventricle and its ability to modify the influence of chronic pulmonary incompetence. ${ }^{10}$ Restrictive $\mathrm{RV}$ physiology has been shown to predict slow postoperative recovery in infants at the time of primary repair. ${ }^{10}$ In a study of the same patients at midterm follow-up, the strongest predictor of restrictive RV physiology was restrictive physiology in the postoperative period. ${ }^{11}$ The implication of restrictive physiology is that the diastolic compliance of the right ventricle is reduced. Therefore the stiff and poorly compliant right ventricle will not react in the same way to the potential chronic overload of pulmonary regurgitation. $\mathrm{Al}-$ though the reaction of the normal right ventricle is progressive dilation in response to pulmonary regurgitation, this normal physiology is lost when the right ventricle is restrictive. ${ }^{12}$ Although this appears to be disadvantageous in the early postoperative period, restrictive physiology has many potential advantages during late postoperative follow-up. In a study of late survivors, restrictive physiology was associated with a smaller heart on chest radiographic analysis, improved exercise function on formal treadmill testing, and a shorter QRS duration on the resting electrocardiogram. ${ }^{13}$

Contemporary patients often undergo primary repair at presentation or when they become symptomatic. ${ }^{14,15}$ Along with abolishing cyanosis and promoting pulmonary artery growth, another stated aim of ever earlier surgical repair has been the preservation of RV performance. However, they might, paradoxically, be exposed to a much greater risk of the effects of pulmonary incompetence than the earlier cohorts and operated on much later in life and with much more intrinsic and postoperative diastolic disease. ${ }^{7}$ With this in mind, there might indeed be some benefit in delaying the primary repair in some patients.

\section{CONCLUSION}

Many questions still remain, and unfortunately, the results of our current treatment strategy will not be realized for many years. However, by identifying the risk factors that affect the progression of right heart failure from pulmonary insufficiency, hopefully we can begin to affect change to gradually lengthen the interval between pulmonary valve disruption and pulmonary valve replacement.

\section{References}

1. Geva T. Indications and timing of pulmonary valve replacement after tetralogy of Fallot repair. Semin Thorac Cardiovasc Surg Pediatr Card Surg Ann. 2006;9:11-22.

2. Bouzas B, Kilner p, Gatzoulis M. Pulmonary regurgitation: not a benign lesion. Eur Heart J. 2005;26:433-9.

3. Bleumink GS, Schut AF, Sturkenboom MC, Deckers JW, van Duijn CM, Stricker BH. Genetic polymorphisms and heart failure. Genet Med. 2004;6:465-74

4. Pasotti M, Repetto A, Tavazzi L, Arbustini E. Genetic predisposition to heart failure. Med Clin North Am. 2004;88:1173-92.

5. Sunakawa A, Shirotani H, Yokoyama T, Oku H. Factors affecting biventricular function following surgical repair of Tetralogy of Fallot. Jpn Circ J. 1988;52:401-10.

6. Chaturvedi RR, Kilner PJ, White PA, Bishop A, Szwarc R, Redington AN. Increased airway pressure and simulated branch pulmonary artery stenosis increase 
pulmonary regurgitation after repair of tetralogy of Fallot. Real time analysis with a conductance catheter technique. Circulation. 1997;95:643-9.

7. Redington A. Physiopathology of right ventricular failure. Semin Thorac Cardiovasc Surg Pediatr Card Surg Ann. 2006;9:3-10.

8. Gatzoulis M. Tetralogy of Fallot. In: Gatzoulis MA, Webb GD, Daubeney PE, eds. Diagnosis and management of adult congenital heart disease. Edinburgh: Churchill Livingston; 2003.

9. Gatzoulis MA, Balaji S, Webber SA, Siu SC, Hokanson JS, Poile C, et al. Risk factors for arrhythmia and sudden death late after repair of tetralogy of Fallot: a mutilcentre study. Lancet. 2000;356:975-81.

10. Cullen S, Shore D, Redington A. Characterization of right ventricular diastolic performance after complete repair of tetralogy of Fallot. Restrictive physiology predicts slow postoperative recovery. Circulation. 1997;95:643-9.

11. Norgard G, Gatzoulis MA, Josen M, Cullen S, Redington AN. Does restrictive right ventricular physiology in the early postoperative period predict subsequent right ventricular restriction after repair of tetralogy of Fallot? Heart. 1998;79: 481-4.

12. Shimazaki Y, Blackstone EH, Kirklin JW. The natural history of isolated congenital pulmonary valve incompetence: surgical implications. Thorac Cardiovasc Surg. 1984;32:257-9.

13. Gatzoulis MA, Clark AI, Cullen S, Newman CG, Redington AN. Right ventricular diastolic function 15-35 years after repair of tetralogy of Fallot. Restrictive physiology predicts superior exercise performance. Circulation. 1995;91: 1775-81.

14. DiDonata RM, Jonas RA, Lang P, Rome JJ, Mayer JE, Castaneda AR. Neonatal repair of tetralogy of Fallot with and without pulmonary atresia. J Thorac Cardiovasc Surg. 1991;101:126-37.

15. Reddy VM, Liddicoat JR, McElhinney DB, Brook MM, Stanger P, Hanley FL. Routine repair of tetralogy of Fallot in neonates and infants less than three months of age. Ann Thorac Surg. 1995;60(suppl):S592-6. 